\title{
Tratamiento de la dislipemia: un reto con implicación multidisciplinar
}

\author{
Treatment of dyslipidemia: \\ a challenge with multidisciplinary involvement
}

\author{
Vidal Pardo JI \\ Unidad de Lípidos -S. de Endocrinología \\ Hospital Universitario Lucus Augusti
}

La alta prevalencia de la enfermedad cardiovascular (ECV) y su repercusión mórbida, 18 millones de muertes al año y un número similar de eventos no fatales ${ }^{1}$, justifica que la prevención y el tratamiento de las mismas constituyan uno de los principales retos a los que se enfrenta la medicina en la actualidad. Galicia se encuentra entre las comunidades con mayor tasa de mortalidad cardiovascular en nuestro país.

El papel protagonista que juega la hiperlipemia en la ECV ha sido ampliamente demostrado en numerosos estudios, así como la importancia de su tratamiento tanto en prevención primaria como secundaria. Existen numerosas guías para el manejo de la dislipemia y prevención cardiovascular publicadas por distintas sociedades.

En el presente número se recoge el "Consenso Gallego Multidisciplinar para el uso de hipolipemiantes", avalado por 10 sociedades científicas de Galicia involucradas en el problema. El mero hecho de su realización ya nos parece un esfuerzo digno de elogio, pero tras su lectura se deduce un enorme interés práctico para el enfoque terapéutico de estos pacientes y para la sanidad pública de nuestra comunidad.

De acuerdo con las guías y recomendaciones existentes, la índole y la intensidad de las intervenciones preventivas de la ECV deben ajustarse al nivel de riesgo. En el consenso se recogen las pautas de las guías ESC/EAS y la estimación de riesgo, mediante el sistema SCORE, al igual que lo ha hecho el Comité Español Interdisciplinario de Prevención Cardiovascular. Este posicionamiento nos parece un acierto, frente a la confusión que había creado la del American College of Cardiology/American Heart Association(ACC/AHA) del 2013 y como en el documento se comenta.

Asímismo nos parece muy útil la recopilación de objetivos y pautas de tratamiento en prevención primaria y secundaria y en distintos situaciones agrupadas por patologías, lo cual redundará en un uso más racional de los fármacos con beneficio para el paciente y para la sostenibilidad del sistema sanitario.

El arsenal terapéutico disponible incluye el tratamiento convencional, basado en estrategias de modificación del estilo de vida y la dieta junto con medicamentos hipolipemiantes, principalmente estatinas. Otros tratamientos disponibles son ezetimiba (combinada con estatinas o en monoterapia en casos de intolerancia a las estatinas o si están contraindicadas), fibratos y secuestradores de ácidos biliares. Existe evidencia con resultados demostrados no solamente en la mejora del patrón lipídico, basada fundamentalmente el la reducción del LDL colesterol, sino que también han demostrado clara reducción de eventos. La mayoría de ellos realizados con estatinas, sin olvidar la aportación del estudio IMPROVE-IT en prevención secundaria con ezetimibe. La elección adecuada del fármaco y la dosis que mejor se adecúe a cada paciente y su perfil lipídico, basada en el coste-beneficio, es una exigencia que no podemos soslayar y en la que nos parece adecuado insistir.

Conviene recordar que el tratamiento de estos pacientes debe realizarse de forma global, considerando al tiempo el control de otras enfermedades y factores de riesgo cardiovascular, frecuentemente asociados. Ello implica el refuerzo en las medidas de modificación de estilo de vida y dieta, no siempre fácil de conseguir.

En la mayoría de los casos son pacientes "polimedicados", en los cuales la adherencia al tratamiento es un problema habitual. Para mejorarla nos parece fundamental el consensuar el tratamiento con el paciente, tras una detallada discusión de los beneficios, costes y riesgos, tanto de las medidas higiénico dietéticas como de los fármacos. Este es un aspecto en el que la NICE hace especial hincapié ${ }^{2}$, en estas y en otra patologías, y con 


\section{"La aparición del presente consenso de las sociedades científicas gallegas constituirá una herramienta útil para el beneficio de los pacientes y para la sostenibilidad del sistema sanitario"}

buenos resultados en la adherencia del paciente. Existen en este campo muchos temas abiertos al debate. Uno de ellos es el de la prevención primaria en sujetos sanos, que viene derivado del amplio uso de estatinas y su efectividad. Muy recientemente se ha publicado el estudio HOPE- $3^{3}$ realizado en pacientes de riesgo intermedio, en prevención primaria y con niveles de lípidos dentro de la normalidad; en él se observa el efecto beneficioso de la reducción de LDL colesterol con una estatina, en la reducción de ECV también en este grupo, en un periodo de seguimiento medio de 5,6 años y consistente con la reducción lograda en poblaciones de riesgo. Si esto debe trasladarse a la clínica diaria es una cuestión que debe asentarse sobre evidencias añadidas y siempre valorando el coste-beneficio y que sea asumible por los sistemas sanitarios.

Otro aspecto a considerar es la dificultad del logro de objetivos en determinadas poblaciones, no solamente en las hipercolesterolemia familiar, lo cual también se ha constatado en nuestro medio ${ }^{4-6}$. Es importante comprender el origen y la magnitud antes de planificar intervenciones adicionales con el fin de alcanzar los niveles objetivos de LDL y reducir la carga de los acontecimientos CV y muertes asociadas.

Para la hipercolesterolemia familiar y en otras muy determinadas situaciones, la FDA y la EMA han aprobado recientemente el uso de fármacos hipolipemiantes de nueva generación (anticuerpos monoclonales inhibidores de la proproteína convertasa subtilisina/kexina de tipo 9, PCSK9), que también han sido autorizados por la administración española (AEMPS). De indudable eficacia demostrada en ensayos clínicos, por las características de los fármacos y el coste, su uso debería restringirse a unidades clínicas especializadas en el manejo de esta patología y tras una rigurosa selección de los casos 7,8 .
En definitiva, para la práctica clínica habitual, podemos obtener importante mejoría en la disminución del riesgo con el arsenal terapéutico convencional, convenientemente utilizado. Un abordaje multidisciplinar, integrado, adaptado a la situación clínica del paciente nos parece el enfoque ideal para las dislipemias.

La aparición del presente consenso de las sociedades científicas gallegas constituirá una herramienta útil para el beneficio de los pacientes y para la sostenibilidad del sistema sanitario, felicitándonos por ello y agradeciendo la iniciativa a los autores.

\section{Bibliografía}

1. GBD 2013 Risk factors collaborators. Global, regional, and national comparative risk assessment of 79 bahavioural, environmental and occupational, and metabolic risks or clusters of risks in 188 countries, 1990-2013: a systematic analysis for the Global Burden of Disease Study2013. Lancet 2015; 386:2287-323.

2. Rabar S, Harker M, O'FlynnN, WierzbickiAS. Guideline Development Group. Lipid modification and cardiovascular risk assessment for the primary and secondary prevention of cardiovascular disease:summary of updated NICE guidance. BMJ 2014; $349 \mathrm{~g} 4356$.

3. Yusuf S, Bosch J, Dagenais G, Zhu J, Xavier D, Liu L, Pais P, López-Jaramillo $P$, Leiter LA, Dans A, Avezum A, Piegas LS, Parkhomenko A, Keltai K, Keltai M, Sliwa K, Peters RJ, Held C, Chazova I, Yusoff K, Lewis BS, Jansky P, Khunti K, Toff WD, Reid $\mathrm{CM}$, Varigos J, Sanchez-Vallejo G, McKelvie R, Pogue J, Jung H, Gao P, Diaz R, Lonn $\mathrm{E}$; HOPE-3 Investigators. Cholesterol Lowering in Intermediate-Risk Persons without Cardiovascular Disease. N Engl J Med. 2016 Apr 2.

4. Pijlman AH, Huijgen R, Verhagen SN, Imholz BP, Liem AH, Kastelein JJ, Abbink EJ, Stalenhoef AF, Visseren FL. Evaluation of cholesterol lowering treatment of patients with familial hypercholesterolemia: a large cross-sectional study in The Netherlands. Atherosclerosis. 2010 Mar;209(1):189-94.

5. Vidal Pardo JI, Pérez Castro TR, López Álvarez XL, García Soidán FJ, Santiago Pérez MI, Muñiz J. Quality of care of patients with type-2 diabetes in Galicia (NW Spain) [OBTEDIGA project]. Int J Clin Pract. 2011;65(10):1067-75.

6. De la Sierra A, Pintó X, Guijarro C, Miranda JL, Callejo D, Cuervo J, Subirà R, Rubio M. Prevalence, Treatment, and Control of Hypercholesterolemia in High Cardiovascular Risk Patients: Evidences from a Systematic Literature Review in Spain. Adv Ther. 2015;32(10):944-61.

7. Documento de Consenso de la SEA (Sociedad Española de Arteriosclerosis) sobre las indicaciones de los Inhibidores de PCSK9. Clinica e Investigacion en Arteriosclerosis. 2016.doc on line.

8. Brown WV, Moriarty PM, McKenney JM. JCL roundtable: PCSK9 inhibitors in clinical practice. J Clin Lipidol. 2016;10(1):5-14. 